\title{
Gemeinsames Bekenntnis zu Qualitätsarbeit in der Medizin
}

\author{
Roxane Küblera, Esther Kraft ${ }^{\mathrm{b}}$ \\ a Operative Leiterin der Schweizerischen Akademie für Qualität in der Medizin SAQM der FMH \\ ${ }^{b}$ Leiterin Abteilung Daten, Demographie und Qualität DDQ der FMH
}

\begin{abstract}
Im Lauf von zwei Jahren hat das Forum Qualität der SAQM eine Qualitäts-Charta erarbeitet. Die Charta hat zum Ziel, die Qualitätsarbeit in der Medizin verbindlich und transparent zu gestalten. Nach einer breit abgestützten Vernehmlassung innerhalb der Ärzteschaft ist die Qualitäts-Charta finalisiert und von 57 Ärzteorganisationen unterzeichnet.
\end{abstract}

\section{Hintergrund}

Die ärztliche Tätigkeit ist geprägt von der Verantwortung gegenüber den Patientinnen und Patienten. Die Qualität der eigenen Leistung gehört als selbstverständlicher Teil zur Arbeit einer jeden Ärztin und eines jeden Arztes und ist zum Nutzen für Patienten, Angehörige und Ärzte. Qualitätsarbeit ist in allen Aspekten zu gewährleisten und zu fördern. Dies ist Bestandteil des medizinisch-ethischen Selbstverständnisses, der Standesordnung der FMH und des Schweizer Rechts ${ }^{1}$. Gesundheits- und gesellschaftspolitische Entwicklungen verlangen nach qualitätsorientierten Kriterien zur Beurteilung der ärztlichen Leistungen. Bezüglich übergeordneter nationaler Qualitätskriterien für die Zulas-

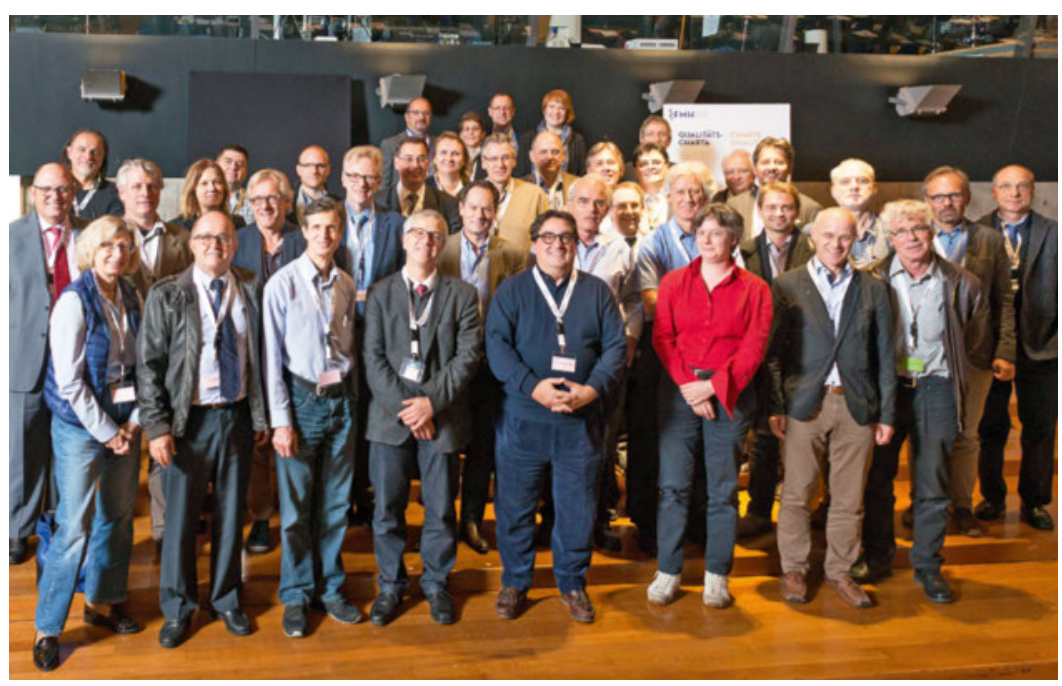

Feierlicher Akt an der Ärztekammer vom 27. Oktober 2016: Vertreterinnen und Vertreter der unterzeichnenden Ärzteorganisationen der Qualitäts-Charta SAQM (Quelle: Frederike Asael). sung für Ärzte hat die FMH bereits klar und wiederholt verbindliche Sprachkompetenzen auf mindestens B2Niveau, eine mindestens dreijährige Tätigkeit an einer schweizerisch anerkannten Weiterbildungsstätte im Fachgebiet der geplanten Niederlassung sowie ein mindestens 5500 Stunden umfassenden Medizinstudium gefordert. Die Fachgesellschaften, die kantonalen Ärzteorganisationen sowie die ärztlichen Dachverbände sind aufgerufen, Qualitäts-Strategien zu entwickeln, zu sichern und einen transparenten Nachweis der erbrachten Qualität zu erarbeiten.

\section{Entstehung}

Die Delegierten und Ersatzdelegierten des Forums Qualität der Schweizer Akademie für Qualität in der Medizin SAQM haben im Lauf von zwei Jahren eine Qualitäts-Charta erarbeitet. Diese wurde bei den in der Ärztekammer vertretenen Organisationen von Januar 2016 bis April 2016 vernehmlasst. Im Juni 2016 verabschiedete das Forum Qualität die finale Version der Charta. Anschliessend hat der Zentralvorstand der FMH die Qualitäts-Charta SAQM gutgeheissen. Die Delegiertenversammlung der FMH wurde über die Qualitäts-Charta im September 2016 informiert. Danach wurden alle in der ÄK vertretenen Organisationen zur Unterzeichnung eingeladen. Die symbolische Unterzeichnung erfolgte im Rahmen der Ärztekammer im Oktober 2016.

\section{Ziele}

Die Qualitäts-Charta SAQM hat folgende Ziele:

- Die Schweizer Ärzteorganisationen bekennen sich gemeinsam zu Qualitäts-Grundprinzipien in den Be- 
Grundprinzipien der Qualitäts-Charta

\begin{tabular}{|c|c|c|}
\hline Transparenz & Verbindlichkeit & Nachhaltigkeit/Korrektheit/Nutzen \\
\hline $\begin{array}{l}\text { Sichtbarmachung von: } \\
\text { A) ärztlichen Aktivitäten zur Гörderung } \\
\text { der Qualität in der Medizin } \\
\text { B) Qualität der ärztlichen } \\
\text { Leistungserbringung }\end{array}$ & $\begin{array}{l}\text { Erstellen einer/s: } \\
\text { C) Qualitätsstrategie } \\
\text { D) Qualitätsbericht }\end{array}$ & $\begin{array}{l}\text { Berücksichtigung von: } \\
\text { E) Nachhaltigkeit } \\
\text { F) ärztliche Aus-, Weiter- und } \\
\text { Fortbildung } \\
\text { G) Gesetzliche und } \\
\text { datenschutzrechtliche Crundlagen } \\
\text { H) Mehrnutzen }\end{array}$ \\
\hline
\end{tabular}

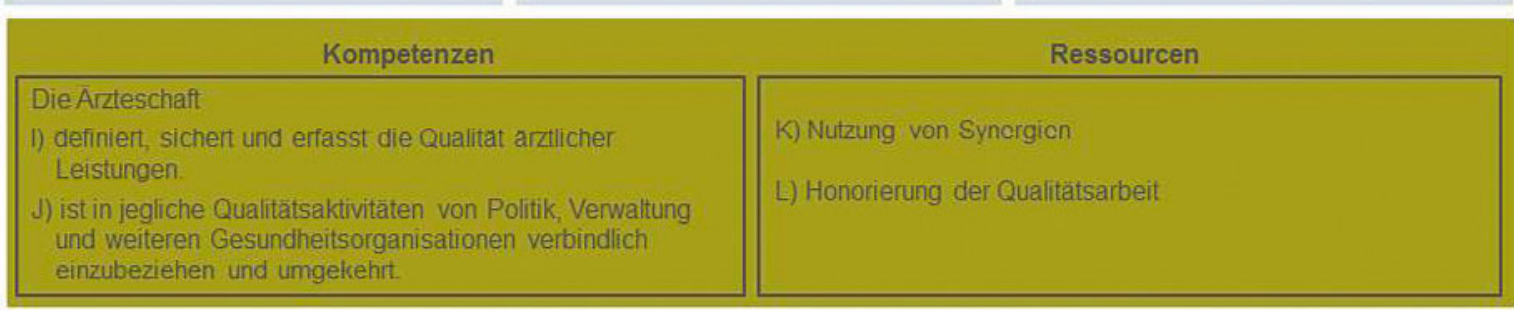

Die Schweizerische Akademie für Qualität in der Medizin SAQM

M) fördert den Zusammenhalt und den Wissenstransfer innerhalb der Ärteschaft zum Thema Qualität und unterstützt die Vernetzung von Qualitătsaktivitāten.

N) ist Triage- und Erstanlaufstelle für alle Belange von Partnern aus Politik, Verwaltung und dem Gesundheitswesen bezüglich der verschiedenen Themen rund um die Qualität der ärztlichen Behandlung.

0) zieht bei eigenen Projekten sowohl betroffene Ärteorganisationen als auch Partnerorganisationen ein, um die fachliche und politische Verankerung frühzeitig zu gewährleisten.

Abbildung 1: Die 15 Grundprinzipien der Qualitäts-Charta SAQM (Quelle: DDQ/SAQM).

reichen Transparenz, Verbindlichkeit, Nachhaltigkeit/Korrektheit, Kompetenzen und Ressourcen.

- Klare Verpflichtungen eingehen, aber auch gemeinsame Forderungen gegenüber der Politik, Verwaltung und weiteren Gesundheitsorganisationen gegen aussen transferieren.

- Vor allem im Bereich der Transparenz der Qualität der ärztlichen Leistungserbringung Verbindlichkeit einführen.

\section{Erstunterzeichnung}

An der Ärztekammer vom 27. Oktober 2016 informierte Dr. med. Christoph Bosshard über die Qualitäts-Charta SAQM. Hierbei betonte er, wie wichtig es für die Ärzteorganisationen ist, sich gemeinsam zu Verbindlichkeit und Transparenz bei der Qualitätsarbeit in der Medizin zu bekennen. Er trug die Namen der unterzeichnenden Organisationen vor und bedankte sich bei ihren Präsidentinnen und Präsidenten für das entgegengebrachte Engagement. Anschliessend lud er sie zur symbolischen Unterzeichnung der Charta ein.

Bereits diese 57 Organisationen haben die QualitätsCharta SAQM unterzeichnet (Stand: 27.10.2016):
- Ärztegesellschaft Baselland

- Ärztegesellschaft des Kantons Luzern

- Ärztegesellschaft des Kantons Schwyz

- Ärztegesellschaft des Kantons St. Gallen

- Ärztegesellschaft des Kantons Zürich

- Ärztegesellschaft Thurgau

- FMCH

- FMP

- FMPP

- Gesellschaft der Ärztinnen und Ärzte des Kantons Solothurn

- Haus- und Kinderärzte Schweiz

- Kollegium für Hausarztmedizin

- Ordine dei Medici del Cantone Ticino

- Schweiz. Ärztegesellschaft für Manuelle Medizin

- Schweiz. Fachgesellschaft für Tropen- und Reisemedizin FMH

- Schweiz. Gesellschaft für Allergologie und Immunologie

- Schweiz. Gesellschaft für Allgemeine Innere Medizin

- Schweiz. Gesellschaft für Anästhesiologie und Reanimation

- Schweiz. Gesellschaft für Arbeitsmedizin 
- Schweiz. Gesellschaft für Chirurgie

- Schweiz. Gesellschaft für Dermatologie und Venerologie

- Schweiz. Gesellschaft für Gastroenterologie

- Schweiz. Gesellschaft für Gefässchirurgie

- Schweiz. Gesellschaft für Gynäkologie und Geburtshilfe

- Schweiz. Gesellschaft für Handchirurgie

- Schweiz. Gesellschaft für Herz- und thorakale Gefässchirurgie

- Schweiz. Gesellschaft für Infektiologie

- Schweiz. Gesellschaft für Kardiologie

- Schweiz. Gesellschaft für Kinder- und Jugendpsychiatrie und -psychotherapie

- Schweiz. Gesellschaft für klinische Pharmakologie und Toxikologie

- Schweiz. Gesellschaft für Medizinische Genetik

- Schweiz. Gesellschaft für Medizinische Onkologie

- Schweiz. Gesellschaft für Neurochirurgie

- Schweiz. Gesellschaft für Nuklearmedizin

- Schweiz. Gesellschaft für ORL, Hals- und Gesichtschirurgie

- Schweiz. Gesellschaft für Pädiatrie

- Schweiz. Gesellschaft für Pathologie

- Schweiz. Gesellschaft für Plastisch-Rekonstruktive und Ästhetische Chirurgie

- Schweiz. Gesellschaft für Pneumologie

- Schweiz. Gesellschaft für Psychiatrie und Psychotherapie
- Schweiz. Gesellschaft für Radiologie

- Schweiz. Gesellschaft für Radio-Onkologie

- Schweiz. Gesellschaft für Rechtsmedizin

- Schweiz. Gesellschaft für Thoraxchirurgie

- Schweiz. Gesellschaft für Urologie

- Schweiz. Neurologische Gesellschaft

- Schweiz. Ophthalmologische Gesellschaft

- Schweizerische Belegärzte-Vereinigung

- Schweizerische Gesellschaft der Vertrauens- und Versicherungsärzte

- Schweizerische Gesellschaft für Notfall- und Rettungsmedizin

- SFSM

- Société Neuchâteloise de Médecine

- Swiss Orthopaedics

- Union Schweizerischer komplementärmedizinischer Ärzteorganisationen

- Unterwaldner Ärztegesellschaft

- VSAO

- Walliser Ärztegesellschaft

Die Unterzeichnung der Qualitäts-Charta ist und bleibt freiwillig und ist auch nach der Ärztekammer weiterhin jederzeit möglich. Kontaktieren Sie uns dazu am besten per E-Mail via saqm[at]fmh.ch.

Mehr Informationen zur Qualitäts-Charta SAQM, Vorlagen zur Qualitäts-Strategie und zum Qualitäts-Bericht finden Sie unter www.saqm.ch $\rightarrow$ Qualitäts-Charta. Dort ist auch eine Fotogalerie von der Live-Unterzeichnung an der Ärztekammer aufgeführt.

Die Schweizerische Gesellschaft für Medizinische Onkologie unterzeichnete als erste Ärzteorganisation die Qualitäts-Charta SAOM. Drei Fragen an ihren Präsidenten, Prof. Dr. med. Markus Maximilian Borner:

Was hat Ihre Gesellschaft hauptsächlich motiviert, die Qualitäts-Charta SAOM zu unterzeichnen?

Die Qualität der onkologischen Versorgung in der Schweiz ist sehr hoch. Dafür sprechen Vergleichszahlen mit dem Ausland - die Vorgaben der strukturierten Weiter- und Fortbildung stellen dies sicher. Der Zeitgeist verlangt aber, dass der Qualitätsnachweis institutionalisiert wird und dabei hilft die Qualitäts-Charta. Ein so wichtiges Anliegen sollte auch aus ökonomischen Gründen zentral angegangen und nicht subsidiär an das schwächste Glied der Versorgungskette heruntergereicht werden.
Verfügung stehen. Es handelt sich um Versorgungsforschung und diese mit Qualitätsfragestellungen zu verknüpfen ist eine grosse Herausforderung, aber auch eine enorme Chance.

Worin sehen Sie die Chancen der Qualitäts-Charta SAQM?

Wenn es der FMH gelingt, einfache und abgestützte Registertools zu implementieren, dann werden wir die notwendigen Instrumente in der Hand haben, die Versorgung ständig bezüglich der Parameter unserer Wahl zu verbessern. Ein ganz wichtiger Punkt ist, dass die Urheber der Charta auch an den Mehraufwand und dessen Vergütung gedacht haben - eine Grundvoraussetzung für die Leistungserbringer in allen Fachgebieten. 